\title{
ANÁLISE DO ACESSO AOS ARTIGOS DE UMA REVISTA ELETRÔNICA ATRAVÉS DOS LOGS
}

\author{
MS. IVONE JOB
}

Mestre em Ciência da Informação pela Escola de Ciência da Informação da Universidade Federal de Minas Gerais (ECI/UFMG), Bibliotecária da Escola de Educação Física da Universidade Federal do Rio Grande do Sul (ESEF/UFRGS) (Porto Alegre - Rio Grande do Sul - Brasil)

E-mail: ivone.job@ufrgs.br

\section{DRA. ANA MARIA MATTOS}

Doutora em Ciência da Informação pela Escola de Ciência da Informação da Universidade Federal de Minas Gerais (ECI/UFMG), Bibliotecária da Escola de Administração da Universidade Federal do Rio

Grande do Sul (EA/UFRGS) (Porto Alegre - Rio Grande do Sul - Brasil)

E-mail: ana.mattos@ufrgs.br

\section{MS. ANA GABRIELA CLIPES FERREIRA}

Mestre em Comunicação e Informação pelo Programa de Pós Graduação em Comunicação e Informação da Universidade Federal do Rio Grande do Sul (PPGCOM/UFRGS), Bibliotecária da Faculdade de Educação da Universidade Federal do Rio Grande do Sul (FACED/UFRGS) (Porto Alegre - Rio Grande do Sul - Brasil)

E-mail: anaclipes@ufrgs.br

\section{RESUMO}

O artigo analisa o acesso aos artigos publicados pela revista Movimento, através do estudo de seus logs. São quantificadas as submissões de artigos e medido o tempo médio de publicação dos artigos aceitos para publicação, de janeiro de 2008 até maio de 2011 . Também são descritas as preferências de acesso dos usuários da revista Movimento em 2010.

PALAVRAS-CHAVE: Comportamento de busca de informação; revistas eletrônicas; análise de logs; bibliometria. 
O uso da revista científica em formato eletrônico é uma rotina nos atuais hábitos dos pesquisadores, principalmente pela diversidade de títulos com acesso aberto, um dos requisitos primordiais para a visibilidade e acessibilidade da publicação (PACKER; MENEGHINI, 2006).

Björk et al. (20 I 0) apresentam a situação do acesso aberto no ano de 2009, ao analisar 1.837 artigos selecionados aleatoriamente da base de dados Elsevier. Desses artigos, 8,5\% ofereciam acesso aberto através dos sites das revistas; 11 ,9\% através dos sites dos próprios autores ou em outros endereços; e 25\% estavam disponíveis gratuitamente para o leitor graças ao pagamento realizado pelos autores para as revistas disponibilizarem seus artigos nesse formato. $\bigcirc$ percentual restante, ou seja, quase 55\% dos artigos possuem acesso restrito de alguma forma, seja através do acesso pago às publicações ou as bases de dados.

Moreira (2005) assevera que a gratuidade de acesso a um grande número de artigos e a outros tipos de documentos influencia na maneira como a comunicação científica se organiza, tendo em vista que documentos com acesso aberto são mais facilmente obtidos e, por consequência, mais citados. De acordo com os resultados de Björk et al. (20 l0), as áreas que mais publicam artigos com acesso aberto são as Ciências da Terra e a Matemática.

Odlyzko (2002) destacava que no início dos anos 2000 os papers em Ciência da Computação disponíveis gratuitamente na Internet eram com frequência mais citados que outros disponibilizados em outros formatos, com acesso eletrônico restrito ou impresso. Evans e Reimer (2009) corroboram tais resultados e garantem que o acesso aberto, além de aumentar o número de citações, estimula a participação global da ciência.

Esse incremento no acesso, apesar de não possuir a dimensão que era imaginada, é significativo, em especial nos países em desenvolvimento do hemisfério sul. Esse aumento não ocorre nos países pobres da mesma região, em função do acesso eletrônico limitado e da estrutura precária para atender aos serviços via Internet. (EVANS; REIMER, 2009).

No Brasil, o Instituto Brasileiro de Informação em Ciência e Tecnologia (IBICT) está à frente das iniciativas de difundir o acesso aberto dentro da comunidade científica. A tendência é observada nas universidades de uma maneira geral, com a criação de repositórios de teses e dissertações, portais de periódicos eletrônicos e, mais recentemente, de repositórios institucionais. O objetivo do IBICT (BRASIL, 2009) é que nosso país se estabeleça "[...] como uma sociedade do conhecimento com acesso totalmente livre e gratuito à informação científica". 
Outros dados interessantes fornecidos pelo IBICT (BRASIL, 2009), quanto ao acesso aberto: em repositórios digitais, o Brasil é a $5^{a}$ nação do mundo, possui a $2^{a}$ maior biblioteca de teses e dissertações, e no ranking de publicações periódicas de acesso aberto está em $3^{\circ}$ lugar. Cabe salientar que a customização de softwares de acesso aberto, destinados à produção de revistas, repositórios e bibliotecas começou no início dos anos 1990.

Estas ações realizadas no Brasil vão ao encontro de compromissos assumidos entre os países e as Nações Unidas, a respeito do desenvolvimento da ciência no século XXI,

[...] a cooperação entre os países desenvolvidos e os em desenvolvimento deve ser executada de conformidade com os princípios de acesso pleno e aberto à informação, equidade e benefício mútuo. (A CIÊNCIA..., 2003, p. 35).

De acordo com Packer e Meneghini (2006), existem oito atributos essenciais para a visibilidade das revistas científicas: (i) ter uma sociedade científica, organização, grupo ou empresa editora responsável por sua publicação; (ii) possuir editor responsável e corpo editorial representativo junto à comunidade científica; (iii) assegurar ampla distribuição institucional e geográfica da afiliação dos autores; (iv) ser publicada de forma eletrônica na Internet e com acesso aberto; (v) disponibilizar a publicação em mais de um idioma (um deles preferencialmente em inglês); (vi) estar indexada em vários índices referenciais nacionais e internacionais; (vii) ter números médios de leituras ou de visitas aos artigos acessados periodicamente; e (viii) receber citações e possuir fator de impacto.

Considerando-se todas essas premissas, este trabalho tem por objetivo geral oferecer exemplos de indicadores de submissão e de acesso aos artigos publicados da revista Movimento através dos logs. Cremos que há possibilidades de que esses tipos de indicadores venham a se constituir em dados que representam a difusão das revistas, não só da Movimento como de todas as revistas em formato eletrônico que se preocupem em dar mais visibilidade, principalmente do processo de submissão, à comunidade científica.

Os logs de acesso são os registros de uma conexão feita entre o computador de um usuário e o computador que armazena os arquivos dos artigos das revistas científicas. Essa ferramenta possibilita quantificar os acessos bem como, em alguns casos, se estiver disponível o plugin adequado no sistema, apresentar todos os endereços de Internet protocols (IPs) que acessaram o artigo, o tempo de duração e a origem do acesso entre outros dados. São dados que poderiam ser oferecidos pelos editores para a comunidade de leitores e autores, no sentido de dar mais informações de comportamento do artigo após sua publicação. 
Especificamente objetiva-se: (a) quantificar as submissões de artigos à Movimento num período escolhido aleatoriamente e por ser mais recente, de janeiro de 2008 até maio de 201 l; (b) medir o tempo médio de publicação dos artigos aceitos para publicação na revista Movimento no mesmo período, pretendendo ter um parâmetro para comparações futuras; (c) especificar a tipologia e o assunto dos trabalhos publicados e mais acessados, identificando a preferência de acesso dos usuários da revista.

É necessário esclarecer, antes de tudo, que o acesso a esses dados da pesquisa estão geralmente restritos às equipes editoriais das revistas ou do veículo eletrônico de acesso remoto. Portanto, foram obtidos com a permissão da comissão editorial da revista Movimento. É um olhar dos bastidores de um dos periódicos mais importantes da Educação Física no Brasil, que possibilitam saber como funciona um periódico e quais são as preferências do leitor. São estudos recentes na literatura e podemos citar semelhantes em outras áreas, como os trabalhos de Vanti (2005; 2010) e de Ferreira e Cunha (2008).

O essencial neste tipo de estudo é mostrar, dar visibilidade a outros indicadores de uso de uma revista eletrônica (dowloads, logs, links), diferentes dos usualmente utilizados pelos serviços de avaliação, como índice de citações e fatores de impacto. Aos do primeiro tipo denominamos estudos webométricos, e aos do segundo denominamos genericamente de estudos bibliométricos.

\section{MEDIDAS DE USO}

Assim como número de artigos baixados (downloads), o número de leituras ou de acessos são indicadores de visibilidade das revistas publicadas na Internet. $\bigcirc$ aumento das ligações externas (links) possibilita o aumento desses indicadores. De acordo com Vanti (2005), ligações externas são um dos tipos de link, e nos estudos webométricos os links são os conectores entre quaisquer unidades de informação (páginas web, diretórios, domínios), também chamados de nós. Os outros tipos de links são: inlink, outlink, selflink, co-links e link interno. O link interno é aquele que remete a uma página dentro do mesmo domínio ou unidade de análise e o link externo é aquele que remete a um site fora do domínio ou unidade de análise à qual pertence.

$\operatorname{Vanti}(2010$, p. 190) define visibilidade como o "[...] número de links recebidos pelo site analisado, desconsiderando os links internos ou autolinks". Esse valor é calculado através da utilização do recurso de busca avançada dos mecanismos de busca, ou seja, um recurso que possibilita analisar os resultados da pesquisa de sites e links em um determinado mecanismo de busca. 
Uma das formas de se analisar a receptividade da produção de artigos científicos publicados em revistas eletrônicas é a análise dos logs. Uma das funções mais importantes na análise dos logs é consolidar informações sobre os padrões de comportamento dos usuários tentando responder algumas questões, como por exemplo: qual o tema predominantemente escolhido, quais os anos preferenciais de busca, quantas visitas foram efetuadas, quantas páginas foram vistas, quais os dias da semana de maior uso, se a busca feita pelo usuário foi realizada digitando diretamente no browser da revista ou através de um buscador, quais palavras o usuário utilizou nesta busca e a distribuição geográfica dos usuários. Neste trabalho não esgotaremos essas questões, mas procuramos examinar algumas variáveis, como exemplos do que pode ser analisado, e que serão explicitadas nas próximas seções do artigo.

\section{REVISTAS EM EDUCAÇÃO FÍSICA NO BRASIL}

Numa busca ao portal Webqualis (COORDENAÇÃO, 2009) e nas bases de dados Thomson Reuters, Scopus, Scielo observamos que das revistas de Educação Física publicadas no Brasil há nove títulos nos extratos intermediários (B| e B2), sendo três indexadas na Thomson Reuters (Motriz, Movimento, Revista Brasileira de Medicina do Esporte), quatro títulos na base Scopus (Movimento, Revista Brasileira de Medicina do Esporte, Revista Brasileira de Cineantropometria \& Desenvolvimento Humano, Revista Brasileira de Educação Física da UEM) e sete títulos na base Scielo (Motriz, Revista Brasileira de Medicina do Esporte, Revista Brasileira de Educação Física e Esportes, Revista Brasileira de Atividade Física \& Saúde, Revista Brasileira de Ciências do Esporte, Revista Brasileira de Ciência e Movimento e Revista Brasileira de Cineantropometria \& Desenvolvimento Humano).

Essas classificações e indexações em bases de dados são importantes fontes para várias avaliações, desde fontes financiadoras de projetos, de incentivos à pesquisa até para os pesquisadores que desejam publicar seus trabalhos numa revista com boa difusão, bem classificada e com prestígio.

O Sistema Qualis de Periódicos' foi criado em 1998 e está atrelado à avaliação da pós-graduação gerenciada pela Coordenação de Aperfeiçoamento de Pessoal de Nível Superior (CAPES). Dentre os indicadores adotados para avaliação, os veículos citados pelos programas de pós-graduação são enquadrados em categorias indicativas de qualidade e figuram como fonte de informação relevante para diversos campos do saber. Há três estratos na avaliação das revistas: A, B e C e cada estrato possui subdivisões. Assim, o estrato superior subdivide-se em Al e A2. No estrato

1. Disponível em: http://qualis.capes.gov.br. Acesso em: 20 mar. 201।. 
intermediário estão BI, B2, B3, B4 e B5 e C corresponde ao estrato inferior de avaliação. Esta classificação é efetivada por uma comissão de representantes de cada área. A Educação Física faz parte da área de conhecimento denominada área 21 , ao lado dos cursos de Fisioterapia, Fonoaudiologia e Terapia Ocupacional. Acrescente-se que um mesmo periódico pode estar categorizado em duas ou mais áreas distintas, o que não demonstra inconsistência, mas a representatividade do veículo para cada campo. Atualmente a Educação Física não possui nenhuma revista nacional no estrato superior A, privilégio das publicações estrangeiras.

O sistema Qualis é reavaliado periodicamente por uma comissão representativa da área de conhecimento específica, o que significa dizer que esses conceitos podem ser alterados de tempos em tempos, teoricamente de três em três anos.

A revista Movimento possui Qualis BI em Educação Física; B2 em Administração, Ciências Contábeis e Turismo e Interdisciplinar; B3 em Educação, História e Saúde Coletiva; B4 em Sociologia e Psicologia. É publicada pela Escola de Educação Física da Universidade Federal do Rio Grande do Sul (ESEF/UFRGS) e seu primeiro número foi publicado em setembro de 1994. Possui periodicidade trimestral, publica artigos em português, inglês e espanhol e está disponível nos formatos impresso e eletrônico, utilizando a plataforma do Sistema de Editoração Eletrônica/Open Journal Systems (SEER/OJS). Seu objetivo é publicar pesquisas científicas sobre temas relacionados à Educação Física em interface com as Ciências Humanas e Sociais, em seus aspectos pedagógicos, históricos, políticos e culturais. Como já vimos, está indexada nas bases do ISI (Thomson Reuters), Scopus, mais a Latindex, Redalyc e Lilacs; Sport Discus; CAB Abstracts Health e está disponível no Portal de Periódicos da CAPES.

Muitas críticas são feitas aos sistemas de avaliação, principalmente ao Qualis, porque utiliza critérios internacionais como o fator de impacto e o índice $\mathrm{H}$ para classificar revistas brasileiras. (LOVISOLO, 2007; TANI, 2007; RODRIGUES, 2007). $\mathrm{Na}$ medida em que o critério para o credenciamento de professores doutores nos cursos de mestrado/doutorado brasileiros está fortemente baseado na capacidade de publicação, e a publicação mais valorizada tem sido aquela veiculada em revistas científicas indexadas no Journal Citation Reports (JCR), é possível supor que em um curto espaço de tempo as pesquisas de cunho mais local e das ciências sociais e humanas não terão espaço nas revistas e, possivelmente, muitos artigos de autores brasileiros estarão redigidos em inglês, para serem aceitos por um público maior e em revistas internacionais, que são mais bem cotadas. (JOB; FRAGA; MOLINA NETO, 2008). Na verdade, isso já está ocorrendo, em várias áreas do conhecimento, tendo inclusive revistas brasileiras totalmente publicadas em inglês. 
Em termos gerais, os países em desenvolvimento como o Brasil estão muito aquém das expectativas num ranking mundial da produção científica, mesmo com os avanços obtidos recentemente. E menos ainda as revistas de Educação Física, o que prejudica a classificação da produção científica dos pesquisadores desta subárea em relação às outras da grande área da saúde, segundo a classificação do CNPq.

\section{MATERIAL E MÉTODO}

Este trabalho tem o objetivo de apresentar e analisar as métricas relacionadas aos acessos realizados pelos usuários da revista Movimento: os dados dos logs coletados no dia 16 de julho de 201 I e as submissões realizadas no período de 2008 a 201 I . A escolha dos períodos foi aleatória, visando os dados mais recentes. Ambos revelam duas facetas diferentes e complementares dos hábitos de acesso dos leitores: um representa especificamente o resultado do que já está publicado (os logs) e outro a busca pela publicação, ou seja, a fase anterior, quando os autores submetem seus manuscritos. Esses períodos de análise exemplificam um tipo de coleta de dados que é viável de ser realizada e ampliada nas revistas eletrônicas que utilizam a plataforma SEER/OJS.

Após a coleta, os dados foram tabulados no software Excel da Microsoft. Destaca-se que percentuais para submissões avaliadas por pares podem não somar 100\%, já que itens submetidos novamente podem ser aceitos, rejeitados ou ainda estarem em processo de avaliação ou edição.

O tempo de publicação é calculado pela média, considerando-se da data de envio para o sistema SEER/OJS até sua publicação, a partir da submissão do artigo aceito. Foram analisadas 8 I I submissões realizadas de janeiro de 2008 até maio de 20 I I. Por outro lado, foram avaliados 60 artigos publicados em 2010 , destacando-se os 10 mais acessados, aqui oferecidos também como um exemplo do que pode ser disponibilizado ao público nas páginas da revista eletrônica.

\section{RESULTADOS}

Quantificaram-se as submissões de manuscritos de 2008 até maio de 201 I , visando obter os artigos aceitos e rejeitados, bem como medir o tempo médio de publicação dos artigos aceitos no período (TABELA I). 
Tabela I - Submissões realizadas à revista Movimento de janeiro de 2008 até maio de 201 I

\begin{tabular}{lcccc}
\hline ANO & SUBMISSÕES & $\begin{array}{c}\text { ACEITO } \\
(\%)\end{array}$ & $\begin{array}{c}\text { REJEITADO } \\
(\%)\end{array}$ & $\begin{array}{c}\text { TEMPO DE PUBLICA- } \\
\text { ÇÃO (DIAS) }\end{array}$ \\
\hline 2008 & 191 & 51 & 49 & 253 \\
2009 & 201 & 31 & 69 & 191 \\
2010 & 293 & 30 & 70 & 150 \\
2011 (jan./jul.) & 170 & 35 & 65 & 36 \\
\hline
\end{tabular}

Fonte: Elaborada pelas autoras

Foram realizadas 855 submissões no período. Em 2009 e 20 I 0, o índice de rejeição foi expressivo, cerca de 70\%, apesar de um leve aumento no número de submissões em 2010 . Há uma lógica nestes dados, porque à medida que a revista recebe mais submissões, o processo seletivo é mais rígido. Como a revista publica, em média, 60 artigos por ano, observamos pelos números da tabela acima, que cerca de 30\% é aprovado, e como decorrência o trabalho dos avaliadores (peer review) é muito intenso, pois seu trabalho depende diretamente da quantidade de submissões e manuscritos que lhe são enviados para recomendar ao editor a aprovação ou rejeição.

O tempo entre a submissão e a publicação dos manuscritos vem diminuindo ao longo dos anos estudados, o que é um excelente indicador, assim como aumento do número de artigos enviados para a submissão. Um dos indicadores verificados nas revistas pelas bases de dados mais importantes é o tempo que ocorre entre a submissão do manuscrito e sua decisão. É bom para o autor, que imediatamente tem sua resposta, e para a revista, que sinaliza uma saúde administrativa, dando conta de sua demanda. O trabalho de gestão de revistas tem um excelente aliado nos sistemas de editoração eletrônica, que fornecem todos os passos para agilizar o trabalho da equipe editorial (DELGADO; RUIZ-PÉREZ; JIMÉNEZ-CONTRERAS, 2006).

A fase que depende essencialmente da intervenção humana é a de avaliação por pares. Diríamos que é a fase mais importante dentro do processo editorial, porque certifica a credibilidade do periódico e a qualidade dos artigos. Esse tempo depende daquele que a revista estipula para o avaliador realizar seu parecer e o tempo em que ele realmente entrega o parecer. Esses detalhes podem atrasar a edição do número da revista (KING; TENOPIR, 1998).

Ao se tabular os logs de acesso, foi possível especificar a autoria, a tipologia e o assunto dos trabalhos publicados e mais consultados (acessados), possibilitando descrever a preferência dos usuários da revista Movimento (QUADRO I). 
Quadro I - Dez artigos mais acessados publicados em 2010

\begin{tabular}{|c|c|c|c|}
\hline AUTORES E ARTIGOS & $\begin{array}{c}\mathrm{N}^{\circ} \mathrm{DE} \\
\mathrm{ACESSOS}\end{array}$ & TIPO & ASSUNTO \\
\hline $\begin{array}{l}\text { SOUZA JÚNIOR, Marcílio Barbosa Mendonça de, et } \\
\text { al. A análise de conteúdo como forma de tratamento } \\
\text { dos dados numa pesquisa qualitativa em Educação } \\
\text { Física Escolar. }\end{array}$ & 941 & $\begin{array}{l}\text { Artigo } \\
\text { original }\end{array}$ & $\begin{array}{l}\text { Análise de conteú- } \\
\text { do. Educação Física } \\
\text { Escolar. }\end{array}$ \\
\hline $\begin{array}{l}\text { ALMEIDA, Felipe Quintão; VAZ, Alexandre Fernandez. } \\
\text { Do giro linguístico ao giro ontológico na atividade epis- } \\
\text { temológica em Educação Física. }\end{array}$ & 908 & $\begin{array}{l}\text { Artigo } \\
\text { original }\end{array}$ & Epistemologia \\
\hline $\begin{array}{l}\text { GOMES, Mariana Simões Pimentel; et al. Ensino das } \\
\text { lutas: dos princípios condicionais aos grupos situacionais. }\end{array}$ & 872 & $\begin{array}{l}\text { Artigo } \\
\text { original }\end{array}$ & Lutas \\
\hline $\begin{array}{l}\text { FARIA, Maria Catarina Meirelles; et al. Atividades mo- } \\
\text { toras cotidianas e suas influências no desenvolvimento } \\
\text { de pré-escolares. }\end{array}$ & 869 & $\begin{array}{l}\text { Artigo } \\
\text { original }\end{array}$ & $\begin{array}{l}\text { Atividade motora/ } \\
\text { pré-escolar }\end{array}$ \\
\hline $\begin{array}{l}\text { CARDOSO, Fernando Luiz; et al. Auto-percepção cor- } \\
\text { poral e preferências motoras de praticantes de dança. }\end{array}$ & 828 & $\begin{array}{l}\text { Artigo } \\
\text { original }\end{array}$ & Corpo. Dança. \\
\hline $\begin{array}{l}\text { OLIVEIRA, Alexandre Palma de; et al. Culto ao corpo } \\
\text { e exposição de produtos na mídia especializada em } \\
\text { estética e saúde. }\end{array}$ & 816 & $\begin{array}{l}\text { Artigo } \\
\text { original }\end{array}$ & Corpo. Mídia. \\
\hline $\begin{array}{l}\text { LAZZAROTTI FILHO, Ari; et al. O termo práticas cor- } \\
\text { porais na literatura científica brasileira e sua repercussão } \\
\text { no campo da Educação Física. }\end{array}$ & 754 & $\begin{array}{l}\text { Artigo } \\
\text { original }\end{array}$ & $\begin{array}{l}\text { Prática Corporal. } \\
\text { Literatura Cientifica. }\end{array}$ \\
\hline $\begin{array}{l}\text { SOUZA Juliano de; MARCHI JÚNIOR, Wanderley. Por } \\
\text { uma sociologia reflexiva do esporte: considerações teó- } \\
\text { rico-metodológicas a partir da obra de Pierre Bourdieu. }\end{array}$ & 715 & Ensaio & Sociologia. \\
\hline $\begin{array}{l}\text { MACHADO, Thiago da Silva; et al. As práticas de } \\
\text { desinvestimento pedagógico na educação física escolar. }\end{array}$ & 673 & $\begin{array}{l}\text { Artigo } \\
\text { original }\end{array}$ & $\begin{array}{l}\text { Educação Física } \\
\text { Escolar. Pedagogia }\end{array}$ \\
\hline $\begin{array}{l}\text { REZER, Ricardo. Reflexões didático-pedagógicas acerca } \\
\text { do ensino do esporte no processo de formação de } \\
\text { professores de Educação Física. }\end{array}$ & 652 & Ensaio & $\begin{array}{l}\text { Esporte. Formação } \\
\text { do professor }\end{array}$ \\
\hline
\end{tabular}

Fonte: Elaborada pelas autoras

Há uma preferência de consulta dos usuários da revista Movimento por artigos originais seguidos de ensaios. Mais da metade dos artigos destacados obteve acima de 800 acessos em um ano, e a outra metade ficou em torno dos 700 acessos. A amostra estudada revela as preferências dentro das especificidades. A revista publica cerca de 60 artigos por ano, e os dez mais consultados mostram o interesse dos leitores pelos estudos da educação física escolar, práticas corporais e aspectos pedagógicos do esporte.

Apresentou-se uma breve análise usando como dados os logs, um registro importante para a compreensão da interação e do comportamento de usuários das revistas eletrônicas. Para os editores da revista é um relevante dado em que poderão se basear para incentivar os artigos sobre os temas mais carentes no seu 
público alvo. Para os leitores, autores e demais usuários da revista é uma forma de comunicar e revelar as fases do processo editorial que normalmente não estão visíveis nas revistas.

Sabe-se que apenas os dados quantitativos dos logs não podem ser traduzidos em informações suficientes sobre o comportamento de busca e uso da informação dos indivíduos, pois estes dados não nos permitem saber se os artigos foram lidos, se foram feitos seus downloads ou mesmo se foram citados. Entretanto, os logs têm valor quando analisados no seu contexto. A análise destes dados nos permite conhecer os aspectos destes usuários remotos, e por isso, deve haver algum monitoramento da interação deles com os serviços eletrônicos oferecidos pela revista.

Como sugestão, outras variáveis dos artigos recuperados na pesquisa podem ser amplamente utilizadas pelos editores da revista, como por exemplo, autores mais constantes que publicam nas revistas, tipologia dos artigos por autor, etc.

Os autores e pesquisadores poderiam utilizar como variáveis os temas e artigos mais relevantes publicados, desde que a revista disponibilize esses dados, o que thes permitiria escolher melhor onde publicar, bem como estabelecer comparações entre os vários títulos e autores com os dados obtidos diretamente da fonte publicadora e não os produzidos por intermediadores da informação, como as bases de dados.

\section{CONSIDERAÇÕES FINAIS}

Os resultados apresentaram um panorama de conceituada publicação da subárea da Educação Física, área das Ciências da Saúde, com as principais características do periódico e as tendências de submissão e difusão dos artigos. A análise de logs possibilita verificar os artigos mais acessados. Dados como o número de submissões, rejeição, tempo de resposta dos artigos demonstra como a revista procura, através da avaliação por pares, manter a qualidade dos artigos recebidos e, pelo tempo de resposta, se manter atrativa para os autores e leitores que desejam uma revista que respeite a periodicidade e a qualidade. Essa é certificada pelos referees e pelos editores.

A importância dessas análises através de logs e número de acessos a artigos de revistas se constituem em indicadores muito recentes de avaliação possibilitados por plataformas eletrônicas de edição de revistas. São indicadores da capacidade de difusão da revista, o quanto são lidos seus artigos, e se são capazes de transmitir informação. Para o autor, é muito importante saber que seu artigo é difundido porque quer ser lido pelo maior número possível de leitores.

São, portanto, bons indicadores para conhecer o grau de penetração nas comunidades, e passíveis de serem utilizados e divulgados pela equipe editorial das 
revistas eletrônicas. Lembramos que a principal finalidade de uma revista científica, como meio de comunicação, consiste em transmitir a informação. E o objetivo último é de alcançar a toda sua audiência, isto é, seu público leitor, que está constituído por uma comunidade científica profissional mais ou menos especializada, dependendo de seu âmbito temático e cobertura.

\section{Articles in an Eletronic Journal: Analysis of Access Through the Logs}

ABSTRACT: This paper evaluates and analyzes the submission and the access of journal articles in the journal Movimento, by studying their logs of access. We quantified the submissions of articles and measured the average time for publication of articles accepted for publication from January 2008 to May 2011. We also describe the preferences of the users to access the journal in 2010.

KEYWORDS: Information seeking behavior; electronic journals; Logs analysis; bibliometrics.

\section{Analisis del acceso a los artículos de una revista electronica mediante sus logs}

RESUMEN: El texto analisa los envios online y el acceso a los artículos de la revista Movimento mediante estudio de sus logs y de los tiempos medios para publicación de los artículos aceptados en el período de 2008 hasta 201 I. También se describe las preferencias de los usuários en el acceso de los artículos publicados em Movimento en el año de 2010.

PALABRAS CLAVE: Conducta en la búsqueda de información; revistas electrónicas; análisis de logs; bibliometría.

\section{REFERÊNCIAS}

BJÖRK, B.-C., et al. Open access to the scientific journal literature: situation 2009. PLoS ONE, San Francisco, v. 5, n. 6, jun. 20 I0. Disponível em: http://www.plosone.org/article/ info\%3Adoi\%2F I0. I 37 I\%2Fjournal.pone.00। I273. Acesso em: 20 jun. 201 I.

BRASIL. Ministério da Ciência e Tecnologia. Instituto Brasileiro de Informação em Ciência e Tecnologia. Portal do Sistema Eletrônico de Editoraçao de Revistas. Brasília, 2009. Disponível em: http://seer.ibict.br/. Acesso em: 22 jun. 201 I

A CIÊNCIA para o século XXI: uma nova visão e uma base de ação. Brasília: UNESCO: ABIPTI, 2003.

COORDENAÇÃO DE APERFEIÇOAMENTO DE PESSOAL DE NÍVEL SUPERIOR(CAPES). Documento de área 2009. Brasília, 2009. Disponível em: 
HTTP://QUALIS.CAPES.GOV.BR/ARQUIVOS/AVALIACAONEBQUALIS/CRITERIOS2007_2009/CRITERIOS_QUALIS_2008_2I.PDF. Acesso em: 20 mar. 2011.

COORDENAÇÃO DE APERFEIÇOAMENTO DE PESSOAL DE NIIVEL SUPERIOR(CAPES). Webqualis. Brasília, 2009. Disponível em: http://qualis.capes.gov.br/webqualis/. Acesso em: 20 mar. 2011 .

DELGADO LÓPEZ-COZAR, E.; RUIZ-PÉREZ, R. ; JIMÉNEZ-CONTRERAS, E. La Edición de Revistas Científicas: directrices, criterios y modelos de evaluación. Granada: Universidad de Granada, 2006. Disponível em: http://www.fecyt.es/fecyt/docs/tmp/I 2247I 3823.pdf. Acesso em: 2 nov. 2011 .

EVANS, J. A.; REIMER, J. Open access and global participation in science. Science, Washington, v. 323, n. 5917, p. 1025, feb. 2009.

FERREIRA, S. M.; CUNHA, A. S. Portal Revcom \& Google analytics: acessando a caixa-preta da informação. Em Questão, Porto Alegre, v. 14, n. I, 2008. Disponível em: <http://seer. ufrgs.br/EmQuestao/article/view/2443/3525>. Acesso em: 20 jun. 201 I.

JOB, I; FRAGA, A. B.; MOLINA NETO, V. Invisibilidade das revistas científicas brasileiras de educação física nas bases de dados. Cadernos BAD, Lisboa, n. I, p. I- I6, 2008.

KING, D. W.; TENOPIR, C. A publicação de revistas eletrônicas: economia da produção, distribuição e uso. Ciência da Informação, Brasília, v. 2, n. 27, p. I76-182, maio/ago. 1998.

LOVISOLO, H. Levantando o sarrafo ou dando tiro no pé. Revista Brasileira de Ciências do Esporte, Campinas, v. 29, n. I, p. 23-33, 2007.

MOREIRA, W. Os Colégios virtuais e a nova configuração da comunicação científica. Ciência da Informação, Brasília, v. 34, n. I, p. 57-63, jan./abr. 2005.

ODLYZKO, A. The rapid evolution of scholarly communication. Learned Publishing, London, v. I5, n. I, p. 7-19, jan. 2002.

PACKER, A. L; MENEGHINI, R. Visibilidade da produção científica. In: POBLACION, D. A.; WITTER, G. P.; SILVA, J. F. M. (Org.). Comunicação \& Produção Científica: contexto, indicadores e avaliação. São Paulo: Angellara, 2006. p. 235-260.

RODRIGUES, L. O. C. - Publicar mais, ou melhor? O tamanduá olímpico. Revista Brasileira de Ciências do Esporte, v. 29, n. I, p. 35-48, 2007.

TANI, G. Educação física: por uma política de publicação visando à qualidade dos periódicos. Revista Brasileira de Ciências do Esporte, v. 29, n. I, p. 9-22, 2007.

VANTI, N. Indicadores web e sua aplicação à produção científica disponibilizada em revistas eletrônicas. In: FERREIRA, S. M. S. P.; TARGINO, M. G. (Org.). Acessibilidade e visibilidade de revistas científicas eletrônicas. São Paulo: Ed. SENAC, 2010. p. 175-212. 
VANTI, N.. Os links e os estudos webométricos. Ciência da Informação, Brasília, v. 34, n. I, p. 78-88, 2005.

Recebido em: 31 jul. 201 I

Aprovado em: | out. 201 |

Endereço para correspondência:

Ivone Job

Rua Felizardo 750

Bairro Jardim Botânico

Porto Alegre, RS

CEP: $90690-000$ 